\title{
Transition to chaos in a shell model of turbulence.
}

\author{
L. Biferale ${ }^{1}$, A. Lambert ${ }^{2}$, R. Lima ${ }^{2,3}$ and G. Paladin ${ }^{4}$ \\ 1 Observatoire de Nice, B.P. 229, 06304 Nice Cedex 4, France \\ 2 Centre de Physique Théorique, CNRS - Luminy \\ case 90\%, F-13288 Marseille, Cedex 9, France \\ 3 The Benjamin Levich Institute, The City College of the City University of New York \\ Steinman Hall, 140th St. and Convent av. New York, NY 10031, USA \\ 4 Dipartimento di Fisica, Università dell'Aquila, \\ Via Vetoio, Coppito I-67100 L'Aquila, Italy
}

\begin{abstract}
We study a shell model for the energy cascade in three dimensional turbulence at varying the coefficients of the non-linear terms in such a way that the fundamental symmetries of Navier-Stokes are conserved. When a control parameter $\epsilon$ related to the strength of backward energy transfer is enough small, the dynamical system has a stable fixed point corresponding to the Kolmogorov scaling. This point becomes unstable at $\epsilon=0.3843 \ldots$ where a stable limit cycle appears via a Hopf bifurcation. By using the bi-orthogonal decomposition, the transition to chaos is shown to follow the Ruelle-Takens scenario. For $\epsilon>0.3953$. the dynamical evolution is intermittent with a positive Lyapunov exponent. In this regime, there exists a strange attractor which remains close to the Kolmogorov (now unstable) fixed point, and a local scaling invariance which can be described via a intermittent one-dimensional map.
\end{abstract}




\section{Introduction}

Shell models for the energy cascade in fully developed turbulence were introduced to mimic the Navier-Stokes equations

$$
\partial_{t} \mathbf{u}+(\mathbf{u} \cdot \nabla) \mathbf{u}=-\frac{\nabla P}{\rho}+\nu \Delta \mathbf{u}+\mathbf{f}_{u}
$$

The reason is that, in a turbulent regime, the number of degrees of freedom necessary to describe the flow generated by eq.s (1.1) is enormous since it roughly increases as a power of the Reynolds number, $R e^{9 / 4}$. However, these degrees of freedom probably are organized in a hierarchical way, so that one expects that simplified dynamical systems could be relevant for the description of the scaling invariance. The basic idea of shell models is to consider a discrete set of wavevectors, 'shells', in $k$-space, and to construct an ordinary differential equation on each shell. The form of the coupling terms among the various shells are chosen according the main symmetries of the Navier Stokes equations.

Standard shell models have a relatively small number of degrees of freedom, so that they can be analyzed as a dynamical system [1-5].

The set of ODE are derived under the assumption that the most relevant mechanism for the behaviour of the velocity field, $u$, is given by a cascade transfer from large to small scales.

Among the huge literature existent nowadays on shell models one finds interesting results about the properties of static solutions for the Novikov-Desnynasky model [3,5], or numerical and analytical studies of the GOY (Gladzer, Ohkitani and Yamada) model in the strongly chaotic regime $[1,2,6,8]$. Amazingly enough, there are not detailed studies of the transition to chaos presents in all of these models. The aim of this paper is to fill this gap, by studying this issue in the case of the GOY model. The understanding of the "route to chaos" is of primary importance to enlighten the intermittent character of the dynamics. In fact, we shall see that in the chaotic regime immediately above the transition, we can study the GOY model through a one-dimensional map that captures the main dynamical mechanisms which are of the origin of intermittency. These mechanisms are much more difficult to be analyzed in the fully chaotic regime which is usually considered. However, it is an open problem to understand how much the dynamical intermittency of shell models is a realistic approximation of real turbulence.

The paper is organized as follows:

In section 1 we define the GOY model. In section 2 there is a detailed discussion on the relative importance of the forward to backward transfer of energy. In section 3 we present our numerical results on the transition to chaos, analyzed by using a bi-orthogonal decomposition [7]; in section 4 we introduce an ad-hoc modified GOY model which allows us to perform a detailed analysis of the intermittent properties nearby the transition.

All the numerical integrations presented hereafter are performed by using a (secondorder) slaved Adam-Beshforth scheme [6]. Results for the model with 19 shells have been obtained by choosing a time step of $\delta t=310^{-4}, \nu=10^{-6}, f=510^{-3} \times(1+i)$ and

$k_{0}=0.05$, while for the model with 27 shells: $\delta t=10^{-5}, \nu=10^{-9}, f=510^{-3} \times(1+i)$ and $k_{0}=0.0625$. 


\section{The GOY model}

In shell models the Fourier space is divided in $N$ shells, each shell $k_{n}(n=1,2, . ., N)$ consisting of the wavenumbers with modulus $k$ such that $k_{0} 2^{n}<k<k_{0} 2^{n+1}$. The velocity increments $|u(\ell)-u(x+\ell)|$ on scale $\ell \sim k_{n}^{-1}$ are given by the complex variables $u_{n}$. The evolution equations are obtained according the following criteria:

(a) the linear term for $u_{n}$ is given by $-\nu k_{n}^{2} u_{n}$

(b) the non-linear terms for $u_{n}$ are combination of the form $k_{n} u_{n^{\prime}} u_{n^{\prime \prime}}$

(c) the interactions among shells are local in $k$-space (i.e. $n^{\prime}$ and $n^{\prime \prime}$ are close to $n$ )

(d) in absence of forcing and damping one has conservation of volume in phase space and the conservation of energy $\frac{1}{2} \sum_{n}\left|u_{n}\right|^{2}$.

In the GOY model the shells $n^{\prime}$ and $n^{\prime \prime}$ are nearest and next nearest neighbors of $n$ so that the evolution equations are:

$$
\left(\frac{d}{d t}+\nu k_{n}^{2}\right) u_{n}=i k_{n}\left(a_{n} u_{n+1}^{*} u_{n+2}^{*}+\frac{b_{n}}{2} u_{n-1}^{*} u_{n+1}^{*}+\frac{c_{n}}{4} u_{n-1}^{*} u_{n-2}^{*}\right)+f \delta_{n, 4},
$$

with $n=1, \cdots N$ and boundary conditions

$$
b_{1}=b_{N}=c_{1}=c_{2}=a_{N-1}=a_{N}=0 .
$$

The velocity $u_{n}$ is a complex variable, $\nu$ is the viscosity, and $f$ is an external forcing (here on the fourth mode). The coefficients of the non-linear terms should obey the relation

$$
a_{n}+b_{n+1}+c_{n+2}=0
$$

to satisfy the conservation of $\sum_{n}\left|u_{n}\right|^{2}$ (energy) in the absence of forcing and with $\nu=0$. Moreover, they are defined modulus a multiplicative factor (related to a time rescaling), so that one can fix $a_{n}=1$. As a consequence, the respect of the main symmetries of the Navier Stokes equations still leaves a free parameter $\epsilon$ so that

$$
a_{n}=1 \quad b_{n}=-\epsilon \quad c_{n}=-(1-\epsilon) .
$$

As we will see in the following, the parameter $\epsilon$ plays an important role in defining both static and dynamical properties of the model.

It is important stressing that scaling law of Kolmogorov $\left(u_{n} \sim k_{n}^{-1 / 3}\right)$ is a fixed point of the inviscid unforced evolution equations with $N \rightarrow \infty$ and neglecting the infrared boundary conditions (1.3). In the next sections we show that the Kolmogorov scaling remains a fixed point of the shell model with $N$ shells, forcing and finite viscosity, and plays a key role in the dynamics.

\section{Static and Dynamical properties}

The GOY model has been defined such as to have "Kolmogorov 1941" (K41) staticsolutions in the inviscid $(\nu=0)$ unforced limit and for the number of shells $N \rightarrow \infty$ : 
$u_{n} \sim k_{n}^{-1 / 3}$. Actually, by studying the static properties of the model, it is easy to recognize that there are two infinite sets of static solutions. Solutions belonging to the same set are characterized by possessing the same scaling exponent.

For the GOY model we have the following two possible static behaviours:

(1) Kolmogorov-like: $u_{n}^{K 41}=k_{n}^{-1 / 3} g_{1}(n)$; with $g_{1}(n)$ being any periodic function of period three.

(2) Fluxless-like: $u_{n}^{f l}=k_{n}^{\left(\ln _{2} \frac{|\epsilon-1|}{2}\right) / 3} g_{2}(n)$; where still $g_{2}(n)$ is any periodic function of period three.

As long as one is interested in scaling laws, the presence of superimposed periodic oscillations could seem particularly disappointing. Nevertheless, the existence in the phase space of an infinite manifold K41-like, instead of a single point, will turn out to be relevant for the dynamical properties of the model.

In order to focus only on the power law scaling it is useful to study the static behaviour of the ratios:

$$
q_{n}=u_{n+3} / u_{n}
$$

Let us notice that the same set of observables have already been used to describe some exotic (chaotic) behaviours of the energy cascade in a different class of shell models [10].

In terms of the $q_{n}$ 's, a static and inviscid solution of eqs. (1.2) can be generated by the iterations of the following one-dimensional complex ratio-map:

$$
q_{n}=\frac{\epsilon}{2}+\frac{(1-\epsilon)}{4 q_{n-1}} .
$$

The map (2.2) has two fixed points $q^{K 41}, q^{f l}$ corresponding to the two possible scaling behaviours for the $u_{n}$ 's:

(1) $q^{K 41}=1 / 2 \rightarrow u_{n} \sim u_{n}^{K 41}$

(2) $q^{f l}=\frac{(\epsilon-1)}{2} \rightarrow u_{n} \sim u_{n}^{f l}$

The first fixed point is ultraviolet (UV) stable for $0<\epsilon<2$ and infrared (IR) stable for any other value of $\epsilon$. For the second fixed point the stability properties are, of course, opposite. For UV (IR) stable we mean that the fixed point is asymptotically approached by starting from any initial condition and by iterating the ratio-map (2.2) forward (backward). From a physical point of view, a forward (backward) iteration of the map (2.2) means a static cascade of fluctuations from small (large) scales to large (small) scales. In the GOY model, the UV stability is the relevant one, since one has a direct cascade of energy or of enstropy. As far as the main dynamical mechanism driving the time evolution of eqs. (1.2) is a forward cascade of energy (like in $3 d$ turbulence), that is for $0<\epsilon<2$ we expect that the system spends a relevant fraction of total time nearby the $K 41$-like static solutions. The aim of this paper consists in quantifying this statement.

Let us stress, also, the importance of the parameter $\epsilon$ from a dynamical point of view. To do this, we introduce the the total flux of energy, $\Pi_{n}$, through the $n$-th shell [6]:

$$
\left.\Pi_{n}=\operatorname{Im}\left(k_{n} u_{n} u_{n+1}\left(u_{n+2}+\frac{(1-\epsilon)}{2} u_{n-1}\right)\right)\right) .
$$


Where in (2.3) we have written only the terms coming from the nonlinear transfer of energy. From (2.3) it is reasonable to expect that by increasing the value of $\epsilon$ from 0 to 1 leads to a depletion of the forward transfer of energy (the coefficient in front to the smaller-scales coupling term goes to zero). Indeed, numerical integration of GOY models with $0<\epsilon<1$ have shown that the main dynamical effect is a forward transfer of energy.

On the other hand, by setting, for example, $\epsilon=5 / 4$, the fluxless like point $u_{n} \sim k_{n}^{-1}$ should dominate the dynamics as it is UV stable while the Kolmogorov-like is UV unstable. Numerically, one observes a reversed (backward) transfer of energy. In fact, the dynamics of GOY models with $\epsilon=5 / 4$ describes the direct enstrophy cascade of $2 d$ turbulent flows $[1,11]$. For $2>\epsilon>1$, beside energy, there also exists another conserved quantity: $\Omega_{\alpha}=\sum k_{n}^{\alpha}\left|u_{n}\right|^{2}$ with $\left.\alpha=-\ln _{2}(\epsilon-1)\right)$ and in the corresponding shell model one observes the direct cascade of such generalized enstrophy $\Omega_{\alpha}$.

Expression (2.3) for the flux of energy also clarifies why the static solution $u_{n}^{f l}$ is called "fluxless". Whenever two shells $u_{n+2}$ and $u_{n-1}$ get trapped by this static fixed point the flux throughout the shell $n$ is completely inhibited, i.e. $\Pi_{n}=0$ (a part viscous and forcing terms). As we will see in the following, the presence of dynamical barriers for the forward cascade of energy is considered the main cause of the intermittent nature of the dynamical evolution.

\section{The transition to chaos in the shell model}

In this section we present a study of the dynamical properties of the GOY model in the "forward-energy cascade" range of parameters $(0<\epsilon<1)$.

Up until now, the model has been studied numerically and analytically only for $\epsilon=1 / 2$ $[1,2,6,8]$. In this case, the most striking result is that the scaling exponents $\zeta_{p}$, of the structure functions $\left(<\left|u_{n}\right|^{p}>\sim k_{n}{ }^{-\zeta_{p}}\right)$, are a non-linear function of $p$, indicating the presence of intermittency in the GOY model which can be described by the multifractal approach [12]. Moreover, the values of $\zeta_{p}$ (for $\epsilon=0.5$ ) are very similar to that measured in numerical simulations and experiments on real fluids.

It is an open problem to relate the multifractality in the $3 d$ real space of the energy dissipation to the multifractality of the natural probability measure on the attracting set for the dynamics in the $2 N$ phase space.

However, there is no reason to choose the value $\epsilon=1 / 2$ for the coefficients of eqs (1.2). A large spectrum of different behaviors can arise in the shell model at varying $\epsilon$, the control parameter for the backward flow of the energy in the cascade.

It is remarkable that for $0<\epsilon \leq 0.3843 .$. , there exists a finite-Reynolds number fixed point (with viscosity and forcing different from zero) which is stable and has Kolmogorovlike scaling in the inertial range.

For example, in figs (1a) and (1b) we have plotted the values of the ratios $q_{n}$ at the fixed point obtained from a numerical integration with $\epsilon=0.05$ and $\epsilon=0.37$. Notice, that the numerical solution coincides exactly with the result predicted by the "forward"

iteration of the ratio-map (2.2) in the inertial range (from the forced shell to the beginning of the viscous range). 
It is interesting to remark, also, that the scaling at the fixed point is not exactly Kolmogorov-like $\left(q_{n}=1 / 2 \forall n\right)$ because of the damped-oscillation introduced by the fact that the ratio-map (2.2) does not start exactly at its fixed point. The oscillations are decreasing by increasing $\epsilon$ and for small $\epsilon$ they mask completely the presence of the Kolmogorov scaling unless one considers a much larger number of shells.

This is, obviously, an effect due to the presence of the infrared boundary conditions (1.3) at small $n$ 's. In the last section we will come back on this issue, by showing how to define new infrared boundary conditions which minimize this effect.

By using the numerical algorithm described in appendix 1 it is possible to follow the fixed point (with viscosity and forcing different from zero) and to compute its stability matrix even for values of $\epsilon$ where it is unstable.

To take into account the invariance under rotations of the fixed point, in the following we analyze the modulus $\left|u_{n}\right|$ rather than the complex variable $u_{n}$.

In fig 1c, the Kolmogorov fixed point $\left|u_{n}^{K 41}\right|$ is shown for $\epsilon=0.3$ (stable) and $\epsilon=0.5$ (unstable).

By looking at the eigenvalues of the stability matrix of the fixed point, we have detected a Hopf bifurcation at $\epsilon=0.3843$, since a couple of complex conjugate eigenvalues have real part which passes from negative to positive value. The fixed point thus becomes unstable and a stable limit cycle appears with a period of $T_{1} \approx 90$ natural time units (n.u.).

This limit cycle loose stability at $\epsilon=0.3953$ and for $0.3953<\epsilon<0.398$, the attracting set is a torus. The two periods of rotations $T_{1} \approx 90 \mathrm{n} . \mathrm{u}$. and $T_{2} \approx 8 \mathrm{n} . \mathrm{u}$.. The motion on the torus can be analyzed by the bi-orthogonal decomposition of the signal and one observes that the two rotation periods are incommensurate with a ratio $T_{1} / T_{2}=12.05 \ldots$.

To illustrate the bifurcation mechanism, fig 2 shows the eigenvalues of the stability matrix at $\epsilon=0.396$ (immediately after the first transition) where there is one couple of complex conjugate eigenvalues with positive real part and at $\epsilon=0.396$, (after the second transition) where there are two of such couples.

At $\epsilon=0.398$ there is a third transition to an aperiodic attractor with a positive maximum Lyapunov exponent. The transition to chaos thus seems well described by the Ruelle-Takens scenario.

In fig 3, we show the bi-orthogonal decomposition [7] of a signal of 307.2 natural time units (n.u.) sampled each 0.6 n.u. which has been obtained from a numerical integration with a time-step of $310^{-4}$ n.u.. A Fourier spectrum of the bi-orthogonal decomposition of the signal provides a clear evidence of the passage from one frequency, to two frequencies and then to chaos, see fig $3 \mathrm{~d}$.

At $\epsilon>0.398$, the time evolution of the dissipative system (1.2) is chaotic and confined on a strange attractor in the $2 N$ dimensional phase space. This fact is a strong evidence that the interaction between shells plays a fundamental role in determining the strength of the intermittency, and that the correct symmetries still leave a large freedom to the system.

Let us now add some comments about these different dynamical regimes, as they can be understood by the bi-orthogonal analysis. We refer to [7] for details on this method from which we recall only some notations for the reader's convenience. Let us decompose 
the modulus of the velocity field as

$$
\left|u_{n}(t)\right|=\sum_{k=1}^{N} A_{k} \phi_{k}(n) \psi_{k}(t)
$$

where $A_{1} \geq A_{2} \geq \cdots \geq A_{n}>0$ and the $\phi_{k}, \psi_{k}$ are orthonormal functions. The first set of functions, $\phi_{k}$, the so-called Topos, are the active directions in the configuration space while the $\psi_{k}$, the so-called Chronos, are the corresponding directions in the space of time-series. The set of coefficients $A_{k}$ is the spectrum of the kernel operator associated to the signal $|u|$, and is called kinetic spectrum, in order to distinguish it from the Fourier spectrum.

First, let us notice that, in all the $\epsilon$-range we have studied, the dynamics of $u_{n}(t)$ in the $2 N$ dimensional phase space always evolves in the neighbourhood of the Kolmogorov fixed point $u_{n}^{K 41}$. this is clearly seen from the fact that the first Topos $\phi_{1}$ is equal to $u^{K 41}$, for any value of $\epsilon$ and the orbits stay in a narrow band in the normal direction to $\phi_{1}$ (since $A_{n}<<A_{1}$ for $n \neq 1$ ). All the shell structures that are present are surprisingly stable at varying $\epsilon$. As the first nine Topos are almost independent of $\epsilon$, only some re-ordering occurring in our $\epsilon$ range, we may thus conclude that the system essentially lives in a elongated ellipsoid inside a space of reduced dimension for most of the time. In [7] one can find an explicit estimation of the time spent in that part of the phase space. Nevertheless, the short time spent in the remaining directions of the configuration space is important for the mechanism of energy transfer from large scales to the viscous small scales. This is shown by the fact that only the last Topos, when the latter are ordered by decreasing energy, have support in the direction of the last shells $19>n \geq 16$.

Concerning the inertial range, the most important feature of the dynamics is that it always takes place along the fixed global structures of these shells ("coherent structures"). It never separates the larger Fourier modes from the small ones, that is to say that, during the energy transfer, the inertial shells are simultaneously and coherently excited. It is also easily seen from the shape of the Topos, that the periodicity three in $n$ plays an important role in the organization of the energy transfer, thus supporting the analysis made in sect. 2. More specifically, for $0.384 \leq \epsilon \leq 0.394$, all the dynamics, included the velocity on the forced shell $u_{4}$, is locked by the fundamental frequency of the circle. For larger $\epsilon$-values, up to $\epsilon=0.395$, the shape of the circle is so deformed that a set of new frequencies appears, for which the linear approximation around the fixed point is no longer valid. In this case, the transfer of energy takes the form of a saw-teeth, a phenomenon which is reminiscent of heat transfer observed in experiments of plasma physics [7]. However, due to the lack of smoothness of the orbit, it is possible that the long range simulation is affected by numerical instabilities appearing for these particular values of $\epsilon$ close but smaller than the second bifurcation point $\epsilon=0.3953$. For $\epsilon>0.3953$, thanks to the bifurcation to a torus, the energy transfer is re-organized by the birth of a new frequency, which is able to lock on the harmonics of the old circle. Indeed, the projection of the dynamics onto the planes spanned by one of the topos $\phi$ supported in the infrared region (the first shells) and each of the $\phi$ supported in the inertial range, are circles of quasi-periodic motion, as shown in fig 3 . Notice that the slopes of $\ln A_{k}$, as function of $k$, computed in the "kinetic inertial range" (the part of the kinetic spectrum which is linear in a log-linear plot) varies according the bifurcations. It grows after each bifurcation, leading to a concentration of energy in the 
first structures of the kinetic inertial range and then falls at the new bifurcation, showing a new arrangement for the distribution of the energy inside the kinetic spectrum.

After each of the bifurcations, we also observe a re-ordering of the structures $\phi_{k}, \psi_{k}$, and the energy of the structures with support in the inertial range of the Fourier spectrum always increases. Finally, after the torus become unstable, the slope continuously decreases. This tendency is compatible with the route to chaos followed by the system at increasing $\epsilon$ (also observed in turbulent flows [7]). As shown in [7], bifurcations take place when certain crossing of the eigenvalues are present (degeneracy), giving rise to rotations of the space and time eigendirections in the degenerate eigenspaces.

This is the reason why the entropy of the bi-orthogonal decomposition, defined as

$$
H\left(\left|u_{n}\right|\right)=-\frac{1}{\ln N} \sum_{k} p_{k} \ln p_{k}
$$

where

$$
p_{k}=\frac{\left|A_{k}\right|^{2}}{\sum_{k}\left|A_{k}\right|^{2}}
$$

is a powerful tool for detecting the bifurcations, as one can see in fig 4 . In order to get a good bifurcation diagram, as it essentially concerns the kinetic inertial range, we restrict the sum in (3.2) to the shell range $n_{i} \leq k \leq n_{2}$. Depending of $\epsilon, n_{1}=2$ or 3 whereas $n_{2}$ varies from 10 to 16 in the GOY model with $N=19$ shells. The difference $n_{2}-n_{1}$ grows at increasing $\epsilon$.

The variation of the entropy as function of $\epsilon$ is well understood from the simultaneous occurrence of degeneracy (the increasing the degree of equidistribution of the weights $p_{k}$ ) and from the exponential decay of the $A_{k}$ in the inertial kinetic range. These two phenomena explain the tendency of the entropy to grow and the occurrence of its local maxima or minima.

Our physical interpretation is that when the probability of having a backward energy transfer is not large enough, the system is able to transfer energy in the most efficient way via a non-intermittent cascade. Above the threshold $\epsilon=0.398$ for the transition to chaos, backward transfer are so efficient that they are able to stop this type of transfer. As a consequence the system may charge energy on the first shells. During a charge, one observes a time varying scaling, i.e. the velocity $\left|u_{n}\right| \sim k_{n}^{-h(t)}$ has an 'instantaneous' scaling exponent $h(t)$ which increases from $1 / 3$ toward larger, and more laminar, values. At a certain instant, the variables $\left|u_{n}\right|$ (with $n$ in the inertial range) become so small that viscosity is comparable to non-linear transfer and dissipate energy directly in the inertial range. Then there is a sudden burst which corresponds to a discharge of the energy accumulated in the first modes. This is a completely different way of dissipating energy, which could give origin to multifractality.

The charge-discharge scenario for intermittency has a counterpart in the Lyapunov analysis of the shell model, where only few degrees of freedom seem to be relevant for the chaotic properties of the system. Although the Lyapunov dimension of the attractor (at least at $\epsilon=0.5$ ) is proportional to the total number of shells of the GOY model [1], only few Lyapunov exponents are positive and there is a large fraction of almost zero 
Lyapunov exponents. By an analysis of the Lyapunov eigenvectors, it can be shown that they correspond to marginal degrees of freedom which concentrate on the inertial range of wavenumbers. There are only few degrees of freedom which are chaotic in a very intermittent way. In fact, during the charge, the energy dissipation stays very low, and the instantaneous maximum Lyapunov exponent is almost zero. When there is an energy burst, there is also a large chaoticity burst, i.e. a very large value of the instantaneous maximum Lyapunov exponent, with a localization of the corresponding eigenvector on the dissipative wavenumbers at the end of the inertial range [2]. These results have important physical implications on the predictability problem which have been discussed in ref [9].

The existence of few active degrees of freedom, in a sea of marginal ones, suggests that, at least for the dynamics of some global observables, an appropriate one-dimensional map could capture the essence of the dynamics. To verify this idea, we choose a variable which can be interpreted as the local singularity, or instantaneous scaling exponent of velocity, that is

$$
h(t)=\frac{1}{3} \frac{1}{(N-13)} \sum_{7}^{N-7} \ln _{2}\left|u_{n} / u_{n+3}\right| .
$$

The Kolmogorov scaling corresponds to $h=1 / 3$, and a laminar signal has $h=1$. The choice of the ratio $q_{n}=u_{n+3} / u_{n}$ is intended to minimize the effect of period three oscillation proper of the fixed point structures, taking into account the results of section 2 .

We numerically find that for $\epsilon<0.385$ the local singularity has the constant value $h=1 / 3$ up to an error smaller than $10^{-2}$, as expected.

In figure 5 , one sees that at $\epsilon=0.396$ (the dynamics evolves on a torus) the scaling exponent $h(t)$ has very small oscillations with two characteristic frequencies around $h=$ $1 / 3$.

At increasing $\epsilon$, the signal $h(t)$ become less and less regular, with a broadening of the probability distribution of $h$, as shown respectively in figs $6 \mathrm{a}$ and $6 \mathrm{~b}$ for $\epsilon=0.42$ and figs $7 \mathrm{a}$ and $7 \mathrm{~b}$ for $\epsilon=0.5$

The maximum scaling exponent $h_{\max } \approx 1$ in both cases, while the minimum one, $h_{\min }$, decreases with $\epsilon$. Note that a value $h(t)<1 / 3$ corresponds to a velocity field more singular than the one given by the Kolmogorov scaling. Such a instantaneous scaling exponent is realized during the fast energy burst due to the discharge, while during the charge the $h$ value slowly fluctuates in an almost regular way around $h \approx 1 / 3$ and eventually increases from $h \approx 1 / 3$ up to $h \approx 1$. We can thus hope to describe the most relevant features of the dynamics by looking at the one-dimensional map $h(t+\delta t)$ versus $h(t)$ with an appropriate time delay $\delta t$, which is shown in figs $6 \mathrm{c}$ and $7 \mathrm{c}$ for $\epsilon=0.42$ and $\epsilon=0.5$ It has the typical form of a map of the Pomeau-Manneville type. The channel close to the diagonal is due to the charge periods while the relaminarization corresponds to a fast energy burst (the discharge process) when a small $h(t+\delta t)$ follows a rather large $h(t)$.

A further complication arises since we are dealing with a dynamical system with many degrees of freedom. Roughly speaking, the majority of them acts as a noisy term which induces vertical (temporal) oscillation on on the one-dimensional map. A picture close to the real mechanisms that are present in the model, seems therefore to be a "1.5"dimensional map. This will permit to include, more accurately, the shell-time structure of 
the symmetries that govern the dynamics of the energy transfer. However it is reasonable to expect that their statistical effect on the mean quantities is not very important, at least near the transition to chaos. Therefore, we have studied the two cases $\epsilon=0.42$ (slightly above the transition) and $\epsilon=0.5$ (the usual value for the shell model). One sees that the laminar channel of the $1 \mathrm{~d}$ map becomes fatter at increasing $\epsilon$, but the relaminarization mechanism is robust. As it is well known, the dynamical behavior of $h(t)$ may be very well affected by "random" oscillations of the one-dimensional map $y=h(t+\delta t)$ versus $x=h(t)$, close to the diagonal $x=y$. In particular, this mechanism may also be responsible for the broadening of the probability distribution of the instantaneous scaling exponent $h$ at increasing $\epsilon$. In practice, the presence of many marginal degrees of freedom is revealed by "random" oscillations in the form of the one dimensional map, without consequences for the qualitative picture.

It is an open issue to decide whether such a dynamical mechanism is relevant to describe the intermittency of real turbulent flow.

\section{A modified GOY model}

To quantify the effect of the intermittent "charge-discharge" mechanism on the scaling exponents $\zeta_{p}$ it is essential to have an inertial range as huge as possible and to minimize non-universal effects due to the infrared and ultraviolet boundary conditions. In order to have "ideal" IR boundary conditions we have to slightly modify the equations of motion for the first two shells. In this way, it is possible to oblige the system to develop a scaling behavior also in the infrared region (the first shells) in order to avoid small deviations of the structure functions with respect to the Kolmogorov prediction $\zeta_{p}=p / 3$, which could arise as an artefact of the forcing imposed on the fourth shells and of the infrared boundary conditions (1.3).

To show it, let us define a new GOY model which is exactly equal to the old one but for the following two facts:

(1) the forcing is moved to the first shell,

(2) the parameters of the two equations for $u_{1}$ and $u_{2}$ are changed in the followings:

$$
\begin{gathered}
a_{1} \rightarrow 2-\epsilon ; \quad \text { instead of } a_{1}=1 \text { (old GOY), } \\
\left.b_{2} \rightarrow-1 ; \quad \text { instead of } b_{2}=-\epsilon \text { (old GOY }\right) .
\end{gathered}
$$

By this choice the requirement of energy conservation (1.4) is satisfied, since $a_{1}+b_{2}+c_{3}=0$, and the first two equations of (1.2) become:

$$
\begin{aligned}
& \left(\frac{d}{d t}+\nu k_{1}^{2}\right) u_{1}=i(2-\epsilon) k_{1} u_{2}^{*} u_{3}^{*}+f \\
& \left(\frac{d}{d t}+\nu k_{2}^{2}\right) u_{2}=i k_{2}\left(u_{3}^{*} u_{4}^{*}-\frac{1}{2} u_{3}^{*} u_{1}^{*}\right) .
\end{aligned}
$$


The rationale for the first request is obvious, while the second change allows us to have an inviscid static fixed point which is at the fixed point of the map (2.2) for any $n$ 's, and therefore the scaling of the static solutions is exactly $u_{n+3} / u_{n} \equiv 1 / 2, \forall n$.

For example, an inviscid static solutions will have:

- $(2-\epsilon) k_{1} u_{2}^{*} u_{3}^{*}=-i f$ from the first equation.

- $u_{4}=1 / 2 u_{1} \rightarrow q_{1}=1 / 2$ from the second equation.

Therefore $q_{n}=1 / 2 \forall n$, because the first iteration is already at the UV stable fixed point of the map (2.2).

For such a class of modified GOY model the static solutions have exactly $\zeta_{p}=p / 3$. The dynamical properties are not modified (energy is still conserved if $\nu=f=0$ ) and the transition to chaos follows the same route described above. The advantage is that now we have a scaling behaviour which is not affected from non-universal infrared boundary effect.

If the intermittent mechanism described in the previous section affects the scaling laws we expect that the beginning of the infrared range should be much more sensible to the presence of charging process than the final zone of the inertial range. Indeed, the probability for a shell nearby the viscous range to be uphill with respect to a barrier of energy is evidently minor than that one of a shell nearby the forcing zone. Therefore, small scales are most of the time laminar or Kolmogorov-like, while large scales are most of the time in a charging highly-unstable status.

Looking at the scaling laws immediately after the chaotic transition $(\epsilon=0.42)$, we have found an interesting trend of the structure functions to be dominated by the Kolmogorov scaling by going toward small scales.

To detect a possible changing of slope along the inertial range we have used "local scaling exponents": $\zeta_{p}(n)$ [13]. Local scaling exponents are defined by choosing a fixed length, say 9 shells, over which fitting the scaling behavior of structure functions and then by moving the analyzed range of shells from the infrared region to the dissipation range. With this definition $\zeta_{p}(n)$ means the results of the fit performed on the structure functions of order $p$ in the range of 9 shells centered at shell $m: n-4<m<n+4$.

In figs $8 \mathrm{a}$ and $8 \mathrm{~b}$ we have plotted the results for $\zeta_{1}(n)$ and $\zeta_{8}(n)$. We have used a modified GOY model with 27 shells in order to increase the total length of the inertial range.

From fig.s 8 is possible to see that these "local scaling exponents" become more and more Kolmogorov-like by going toward the viscous range. In order to improve the quality of our fit we have used a technique introduced by Benzi et al. [14] called ExtendedSelf-Similarity (ESS). ESS has proved to be efficient in minimizing finite-size effect and non-universal character in structure functions. The main idea consists in choosing one structure functions as reference and then studying the scaling properties of all other structure functions versus that reference-one.

This trend toward K41 scaling seems to us in agreement with the previous intermittent picture, small scales are dominated by laminar or Kolmogorov scaling, while large scales are most of the time more turbulent then a K41 solutions due to the charging process.

From preliminary data, the same effect seems to be absent for larger values of $\epsilon$ (such as the standard value $\epsilon=0.5$ ) which lead to more chaotic systems. It is an open question whether the same trend would be present, by taking a number of shells large enough. 


\section{Conclusions}

We have studied the transition to chaos in the GOY shell model, at varying the parameter $\epsilon$ related to the strength of backward energy flow. We thus observe the passage from a stable fixed point (corresponding to the Kolmogorov non-intermittent energy cascade) toward a chaotic attractor (corresponding to the intermittent cascade) through the Ruelle-Takens scenario. We provide a numerical evidence that the strange attractor which has a large fractal dimension remains close to the (now unstable) manifold possessing Kolmogorov scaling.

Immediately above the threshold for chaos, we are able to show that the physical mechanism of the intermittency of energy dissipation, is due to a charge-discharge mechanism which can be described by a one-dimensional map. This is a consequence of the presence of few 'active' degrees of freedom while the remaining marginal degrees of freedom (responsible for the high dimensionality of the attractor) have a sort of noisy effect on the one-dimensional map. The map is of the Pomeau-Manneville type where the channel close to the diagonal is related to the charge periods while the relaminarization corresponds to a fast energy burst (the discharge process).

We have also introduced a modified shell model where there is a good scaling behavior even in the infrared (small wave-number) range. The presence of a huge range of scaling shells allows us to study in detail the possible presence of deviations to the usual power law scaling. We find that for the GOY model in the "weak" chaotic region $(\epsilon=0.42)$ the structure functions tend to become Kolmogorov-like by decreasing the analyzed scales. This could be an indication that multifractal corrections disappear in the limit of large Reynolds number, at least for $\epsilon$ slightly above the transition to chaos. It is very difficult to decide by numerical experiments if such an effect is present at the usual value $\epsilon=0.5$, because one should consider very high Reynolds that is a very large number of shells. It still remains an open problem to understand whether the charge-discharge intermittency described in this paper might be compatible with the Kolmogorov scaling laws, or it brakes a global scaling invariance leading to multifractality, as commonly believed on the basis of numerical experiments [2-6] and analytic calculations [8].

Acknowledgments We thank Daniele Carati for many interesting discussions. L. B. was partially supported by a Henri Poincaré fellowship (Centre National de la Recherche Scientifique and Conseil Général des Alpes Maritimes) and by the "Fondazione Angelo della Riccia". G.P. is grateful to the C.P.T. de Luminy for warm hospitality. 


\section{Appendix}

In this appendix we show the numerical algorithm for the search of the Kolmogorov fixed point at $\epsilon>0.3843$ where it is unstable. Let us denote by

$$
\frac{d U}{d t}=F(\epsilon, U)
$$

the system (1.2) considered as a real $2 N$ dimensional system where $U_{n}=\operatorname{Re}\left(u_{n}\right)$ and $U_{n+N}=\operatorname{Im}\left(u_{n}\right)$ with $n=1, \cdots, N$. The point $U^{K 41}(\epsilon)$ is a fixed point of (A1) if $F\left(\epsilon, U^{K 41}\right)=0$.

In order to determine the value of the fixed point for $\epsilon+\delta \epsilon$, we make the observation, which stems from numerical simulations in a range of $\epsilon$-values where the fixed point is stable, that $U^{K 41}(\epsilon)$ moves very slowly with $\epsilon$. We can thus expand up to the first order in $\delta \epsilon$ the relation

$$
F\left(\epsilon+\delta \epsilon, U^{K 41}(\epsilon+\delta \epsilon)\right)=0 .
$$

As $F$ depends linearly on $\epsilon$ we have the exact relation

$$
F\left(\epsilon, U^{K 41}(\epsilon+\delta \epsilon)\right)+\delta \epsilon \frac{\partial F\left(\epsilon, U^{K 41}(\epsilon+\delta \epsilon)\right)}{\partial \epsilon}=0 .
$$

By assuming that

$$
U^{K 41}(\epsilon+\delta \epsilon)=U^{K 41}(\epsilon)+V^{(\epsilon)} \delta \epsilon,
$$

and using the fact that $F\left(\epsilon, U^{K 41}(\epsilon)\right)=0$, one obtains from (A3)

$$
D F\left(\epsilon, U^{K 41}(\epsilon)\right) V^{\epsilon}+\frac{\partial F\left(\epsilon, U^{K 41}(\epsilon)\right)}{\partial \epsilon}=0,
$$

where $D F\left(\epsilon, U^{K 41}(\epsilon)\right)$ is the the stability matrix of the system (1.2) calculated at $U^{K 41}(\epsilon)$. this equation can be solved in $V$ and reads

$$
U^{K 41}(\epsilon+\delta \epsilon)=U^{K 41}(\epsilon)-\delta \epsilon\left[D F\left(\epsilon, U^{K 41}(\epsilon)\right)\right]^{-1} \frac{\partial F\left(\epsilon, U^{K 41}(\epsilon)\right)}{\partial \epsilon} .
$$

The two matrices $D F$ and $\partial F / \partial \epsilon$ are obtained by a direct numerical calculation. In this paper we have iterated $A 5$ with $\delta \epsilon=10^{-4}$, starting from a stable fixed point $U^{K 41} \epsilon_{0}=0.2$ which has been obtained by a long numerical integration of the shell model. The stability matrix $D F$ is then found and diagonalized at the $\epsilon$ 's of interest (see fig.s 2 for $\epsilon=0.386$ and $\epsilon=0.396)$. 


\section{References.}

[1] M. Yamada and K. Okhitani, J. Phys. Soc. of Japan 56, 4210 (1987); Progr. Theo. Phys. 79, 1265 (1988); Phys. Rev. Lett. 60, 983 (1988).

[2] M.H. Jensen, G. Paladin and A. Vulpiani, Phys.Rev.A 43, 798 (1991).

[3] A.M. Obukhov, Atmos. Oceanic. Phys. 7, 41 (1971); A.M. Obukhov, Atmos. Oceanic. Phys. 10, 127 (1974); V.N. Desnyaski and E.A. Novikov, Prikl. Mat. Mekh. 38507 (1974)

[4] E.B. Gledzer, Sov. Phys. Dokl. 18, 216 (1973).

[5] E.D. Siggia, Phys. Rev.A 15, 1730 (1977); E.D. Siggia, Phys. Rev.A 17, 1166 (1978); R.M. Kerr and E.D. Siggia, J. Stat. Phys. 19, 543 (1978); R. Grappin, J. Leorat and A. Pouquet, J. de Physique 47, 1127 (1986). T. Bell and M. Nelkin, Phys. of Fluids 20345 (1977).

[6] D. Pisarenko, L. Biferale, D. Courvasier, U. Frisch and M. Vergassola Phys. of Fluids A5, 2533 (1993).

[7] N. Aubry, R. Guyonnet and R. Lima, J. Stat. Phys. 64 n. $3 / 4$ (1991); ibidem 67 n. 1/2 (1992); Th. Dudok de Wit, R. Lima, A.-L. Pecquet, J.-C. Vallet to appear in Phys. of Fluids B (1994); N. Aubry, F. Carbone, R. Lima and S. Slimani to appear in J. Stat. Phys. (1994)

[8] R. Benzi, L. Biferale and G. Parisi, Physica D65, 163 (993)

[9] A. Crisanti, M.H. Jensen, G. Paladin and A. Vulpiani, Phys. Rev. Lett. 70, 166 (1993).

[10] L. Biferale, M. Blank and U. Frisch, to appear in J. Stat. Phys. (1994).

[11] P. Frick and E. Aurell, Europhys. Lett. 24725 (1993).

[12] G. Paladin and A. Vulpiani Phys. Rep. 156, 147 (1987).

[13] S. Grossmann and D. Lohse, "Universality in fully developed turbulence", Preprint of the University of Chicago, (1993).

[14] R. Benzi, S. Ciliberto, C. Baudet, R. Tripiccione, F. Massaioli and S. Succi, Phys. Rev. E, 48 R29 (1993). 


\section{FIGURE CAPTIONS}

Fig 1a Values of the ratios $q_{n}$ at the fixed point of equations (1.2) with $\epsilon=0.05$ and with superimposed the values predicted by the ratio-map (2.2). Circles are the outputs from the numerical integration, where squares correspond to the ratio-map values. The straight line correspond to the exact K41 scaling $\left(q_{n}=1 / 2 \forall n\right)$

Fig $1 \mathrm{~b}$ The same as in figure (1a) but with $\epsilon=0.37$.

Fig 1c Kolmogorov fixed point $\ln _{2}\left|u^{K 41}{ }_{n}\right|$ versus $n$ for $\epsilon=0.3$ (dashed line) and $\epsilon=0.5$ (solid line).

Fig 1d Topos $\phi_{1}$ versus $n$ for $\epsilon=0.3$ (dashed line) and $\epsilon=0.5$ (solid line), given by the biorthogonal decomposition of a signal obtained from a numerical integration of 307.2 n.u. after a transient of 3900 n.u. starting from an initial condition close to the Kolmogorov fixed point. These initial conditions are also used to obtain figs 4, 5, 6 and 7 .

Fig 2 Imaginary versus real part of the eigenvalues of the stability matrix of the Kolmogorov fixed point at $\epsilon=0.386$ (limit cycle) and at $\epsilon=0.396$ (torus).

Fig 3 Bi-orthogonal decomposition of a signal obtained from a numerical integration of 307.2 n.u. after a transient of 3900 n.u.. In figs $3 \mathrm{a}, 3 \mathrm{~b}$ and $3 \mathrm{c}$ the time unit of the horizontal axis is 0.6 n.u..

Fig 3a Three-dimensional plot of the torus obtained by the Chronos $\psi_{8}, \psi_{9}$ and $\psi_{3}$.

Fig 3b Oscillations of Chronos $\psi_{3}(t)$ with period $T_{1} \approx 90$ n.u..

Fig $3 \mathrm{c}$ Oscillations of Chronos $\psi_{9}(t)$ with period $T_{2} \approx 8$ n.u., modulated by the first harmonics of period $T_{1}$

Fig 3d Semi-log plot of the Fourier power spectrum of Chronos $\psi_{9}$.

Fig 4 Entropy of the bi-orthogonal decomposition versus $\epsilon$ where $n_{1}=3$ and $n_{2}=N=19$. For each point, the entropy is obtained from a numerical integration of 307.2 n.u. after a transient of 6000 n.u. starting at the corresponding Kolmogorov fixed point $u^{K 41}(\epsilon)$. These initial conditions are also used to obtain figs 1d, 5, 6 and 7.

Fig 5 Instantaneous scaling exponent $s(t)=3 h(t)$ as function of time at $\epsilon=0.396$ (torus). The Kolmogorov scaling corresponds to $s=1$.

Fig 6a Instantaneous scaling exponent $s(t)=3 h(t)$ as function of time, at $\epsilon=0.42$. Note that a laminar velocity field has $s=3$, and the Kolmogorov fixed point $s=1$.

Fig 6b Probability distribution of the instantaneous scaling exponent $s=3 h$ at $\epsilon=0.42$.

Fig 6c One-dimensional map obtained by plotting $s(t+\delta t)$ versus $s(t)$ with $s(t)=3 h(t)$, $\delta t=0.6$ n.u. for $\epsilon=0.42$.

Fig 7a Instantaneous singularity $s(t)=3 h(t)$ as function of time at $\epsilon=0.5$.

Fig 7b Probability distribution of the instantaneous singularity $s=3 h$ at $\epsilon=0.5$.

Fig 7c One-dimensional map obtained by plotting $s(t+\delta t)$ versus $s(t)=3 h(t)$ with $\delta t=0.6$ n.u. for $\epsilon=0.5$.

Fig 8a Local scaling exponent for structure function of order 1 . Notice the trend toward the K41 value: $\zeta_{1}=1 / 3$ by decreasing the set of analyzed scales in the inertial range.

Fig $8 \mathrm{~b}$ The same as in figure (8a) but for the structure functions of order 8 (here the $\mathrm{k} 41$ 
value corresponds to $\zeta_{8}=8 / 3=2.666$..). 